\title{
An Optimized Feature Regularization in Boosted Decision Tree
}

\author{
Ravichandran, Krishna Mohanta, C.Nalini
}

\begin{abstract}
We put forward a tree regularization, which empowers numerous tree models to do feature collection effectively. The type thought of the regularization system be to punish choosing another feature intended for split when its gain is like the features utilized in past splits. This paper utilized standard data set as unique discrete test data, and the entropy and information gain of each trait of the data was determined to actualize the classification of data. Boosted DT are between the most prominent learning systems being used nowadays. Likewise, this paper accomplished an optimized structure of the decision tree, which is streamlined for improving the efficiency of the algorithm on the reason of guaranteeing low error rate which was at a similar dimension as other classification algorithms
\end{abstract}

Key word-Decision Tree, Boosting, Regularization, Feature optimization

\section{INTRODUCTION}

We suggest tree-regularization structure designed for feature selection in Decision Trees (DT). The regularization system abstains from choosing another feature used for split the data in tree hub while so as to feature deliverscomparative gain to features officially chose, and therefore creates a minimal feature sub-set. The regularization-system just need solitary replica to be manufactured, and can be effectively added to a wide scope of tree-based models which utilize one feature for split datapoints at a hub. We actualized the regularization structure on arbitrary woods [2] and boosted trees [3]. Investigations show the viability and competence of two regularized treeensembles. As tree-models normally handle all out and mathematical factors, missing qualities, diverse balance among factors, associations and nonlinearities and so forth., the tree regularization system gives a successful and productive feature selection answer for some down to earth issues. Boosting is a standout amongst the most well known learning systems being used today, joining numerous powerless students to shape a solitary solid on. Shallow decision trees are regularly utilized as frail students because of their effortlessness and heartiness by and by. This ground-breaking mix is the learning spine behind many best in class techniques over an assortment of areas, for example, computer vision, conduct examination, and record ranking to give some examples, with the additional advantage of displaying quick speed at test time. Learning speed is significant also. In dynamic or ongoing learning circumstances, for example, for human-on the up and up procedures or when managing data streams, classifiers must adapt rapidly to be viable. This is our inspiration: quick

Revised Manuscript Received on April 12, 2019.

Ravichandran, Research scholar, BIHER.

Dr.KrishnaMohanta,Associate Professor, Kakatiya Institute of Technology and Science, Dept. of CSE. Higher Education and Research.(E-mail: meravicse@gmail.com)
Dr.C.Nalini,Professor, Department of CSE ,Bharath Institute of

training without giving up accuracy. To this end, we propose a principled methodology. Our technique offers a speedup of a request of extent over earlier methodologies while keeping up indistinguishable execution. The commitments of our work are the accompanying: Given the execution on a subset of data, we demonstrate a bound on a stump's classification error, information gain, Gini polluting influence, and change. In light of this bound, we propose a algorithm ensured to create indistinguishable trees as traditional algorithms, and tentatively show it to be one request of size quicker for classification errands. We layouta algorithm for rapidly Boosting decision trees utilizing our brisk treetraining technique, material to any variation of Boosting. In the accompanying areas, we talk about related work, examine the tree-boosting process, depict our algorithm, demonstrate our bound, and finish up with investigations on a few datasets, exhibiting our gains.

Section 2 describes related work section. Section 3 proposes the tree regularizationframework,theregularizedrandomforestand the regularized-boosted RT. Section-4 the evaluation criterion for feature-selection. Section-5 concludes this work.

\section{RELATED WORKS}

Feature selection strategies can be separated into filters, wrappers and implanted techniques [5]. Filter choose features dependent on criterion autonomous of some administered student $[6,7]$. In this manner, the execution of filters may not be ideal for a picked student. Wrappers utilize a student as black-box to assess the overall convenience of a feature sub-set [8]. Wrappers scan the best feature subset for a given administered student; be that as it may, wrappers will in general be computationally costly [9]. Rather than regarding a student as a black box, implanted techniques select features utilizing the information got from training a student.. At every cycle, SVM-RFE takes out the feature with the littlest weight acquired from a prepared SVM. The system can be reached out to classifiers ready to give variable importance scores, for example tree models [11]. The wrappers and installed strategies presented above require building different models, for example RFE system [10] require fabricating possibly models. Indeed, even to the detriment of some worthy misfortune in forecast execution, it is entirely alluring to create feature selection strategies that just need guidance a solitary model which may extensively diminish the training time [5]. The treeregularization system planned now empowers numerous sorts of decision tree models to perform feature subset 
selection by structure the models just a single time. While tree model are prominently utilized for DM, the tree regularization structure gives a compelling and productive answer for some pragmatic issues.

\section{FEATURE REGULARIZATION DECISION TREE (FRDT)}

\subsection{Feature selection}

From the computational perspective, the most proficient one is to make feature ranking based on the greatest SC measure esteems determined for every one of the features, for the entire training dataset. The expense is equivalent to while making decision stubs. In any case, when the classification task is a multiclass one, separabilities of single splits can not mirror the genuine righteousness of the features. Sometimes a few splits might be important to demonstrate that the feature can accurately separate diverse classes. In this way it is sensible to reward such features, however some punishment must be presented, when different splits are being examined. These thoughts brought the accompanying algorithm:

Algorithm 1 FeatureAnalysis
Input: Training data
Output: Features of importance.
1:For each feature f
$\begin{array}{lc}\text { 2: } & \mathrm{T} \leftarrow \mathrm{SC} \text { tree } \\ 3: & \mathrm{n} \leftarrow \text { leaves in } \mathrm{T} . \\ \text { 4: } & \text { For } \mathrm{i}=\mathrm{n}-1 \text { down to } 1 \\ \text { 5: } & \text { si } \leftarrow \mathrm{SC}(\mathrm{T}) \\ \text { 6: } & \text { Prune } \mathrm{T} \text { by deleting a node } \mathrm{N} . \\ \text { 7: } & \text { Define the rank } \mathrm{R}(\mathrm{f}) \\ \text { 8:Return the list of features } \mathrm{R}(\mathrm{f}) .\end{array}$

This executes a full-featured" filter the decision tree building algorithm chooses the splits locally, for example as for the splits chose in before stages, with the goal that the features happening in the tree, are integral. It is essential to see that in spite of this, the computational multifaceted nature of the algorithm is appealing. At times the full classification trees utilize just a little piece of the features. It doesn't permit choose any number of features the most extreme is the quantity of features utilized by the tree, on the grounds that the algorithm give no in order concerning the ranking of the remainder of features.

\subsection{Regularized trees}

A littler produces a bigger punishment to a feature not having a place with $F$. A tree-model utilizing the treeregularization system is known as a regularized tree model. A regularized tree model consecutively adds new features to $F$ if those features give significantly new prescient in sequence concerning $\mathrm{Y}$. The $\mathrm{F}$ as of a manufactured regularize tree-model is relied upon to contain a set of useful, however non-repetitive features. At this time $\mathrm{F}$ gives the chose features straightforwardly, which has the favorable position greater than a feature status technique in which a subsequent selection rule should be connected.
Algorithm 2 Regularized Tree
1: for $\mathrm{m}=1: \mathrm{M}$ do
2: $\quad \operatorname{gain}_{\mathrm{R}}\left(\mathrm{X}_{\mathrm{m}}\right)=0$
3: $\quad$ calculatethegain ${ }_{\mathrm{R}}$ forallvariablesinF
4: $\quad$ penalize using newfeatures

\author{
5: end \\ 6: if gain $=0$ then leaf and return $\mathrm{F}$ end if \\ 7: divide data into $\gamma$ nodes by $\mathrm{X}$ \\ 8: return $\mathrm{F}$
}

The arbitrary tree arbitrarily chooses and tests $\mathrm{K}$ factors out of $\mathrm{M}$ factors at each and recursively splits data utilizing the information gain paradigm. The irregular tree utilizing the regularization structure is known as the regularized arbitrary tree algorithm which is appeared in Algorithm 2. The algorithm centers around delineating the tree regularization system and discards a few subtleties not legitimately significant to the regularization structure.

\subsection{Optimization of Decision Tree}

The primary structure ofa decision tree dependent on FRDT algorithm. This paper analyzed the consequences of Regional division determined by the decision tree algorithm and Bayes classifier for a similar date set .The partitioned area speaks to the classification aftereffects of the algorithm in the given facilitate district. The test results illustrate the distinction between the FRDT decision tree algorithm and Bayes classifier. Adjacent to two referenced algorithms, this paper utilized K-mean algorithm to group the standard data set fisheriris to investigate their separate Classification feature. The simulation results demonstrate that the FRDT decision tree algorithm can accurately mirror the attributes of the data, which not just has phenomenal capacity of classification and prediction, yet can without much of a stretch generate the guidelines. In any case, amid the procedure of classification, The FRDT algorithm will in general pick the attributes with progressively same incentive to generate the decision tree, which might be not constantly ideal. As a rule, we ought to pick the more basic structure if decision tree on the reason of the base of the classification error's number. With the extending of decision tree, the Proportion of classification error will step by step decrease, ut the number will increment in the meantime

\subsection{Boosting tree}

A boosted classifier can be prepared by avariciously limiting a misfortune work, for example by enhancing scalar and frail student at every emphasis. Prior to training starts, every datum test is appointed a non-negative weight. After every cycle, misclassified samples are weighted all the more vigorously in this way expanding the seriousness of misclassifying them in following emphases. Notwithstanding the kind of Boosting utilized, every cycle requires training another powerless student given the example weights. We center on the situation when the powerless students are shallow trees. The ideal feature can regularly be assessed at a small amount of the computational cost utilizing the accompanying heuristic this heuristic does not ensure to restore the ideal feature. Notwithstanding, in the event that we were some way or another ready to bound the error, we would almost certainly prune features that would presumably underachieve. Appropriately, we propose another technique dependent on looking at feature execution on subsets of data, and thusly, pruning underachieving 
features. Choosing which timetable of $\mathrm{m}$-subsets to utilize is a nuance that requires further clarification. In spite of the fact that this decision does not influence the optimality of the prepared stump, it might impact speedup. In the event that the principal "generally little" $\mathrm{m}$-subset is excessively little, we may miss out on low-error features prompting less brutal pruning. In the event that it is excessively huge, we might do superfluous algorithm. Moreover, since the estimation of fundamental error incurs some computational cost, it is unrealistic to utilize each $\mathrm{m}$ when training on dynamically bigger subsets. Utilizing our fast stump training strategy, we decide the ideal parameters without thinking about each example for each feature. By pruning underachieving features, a great deal of algorithm is spared.

\section{RESULT:}

Data accumulation and preprocessing are the underlying phases of the data mining process. Since just legitimate data will create accurate yield, data preprocessing is the key stage. For this examination, we utilize the Weather data from UCI. We simply think about related information and disregard the rest. So as to check the prescient capacity of the precipitation prediction model dependent on the proposed FRDT algorithm, the model is contrasted and the conventional C5.0 and CART precipitation prediction model.

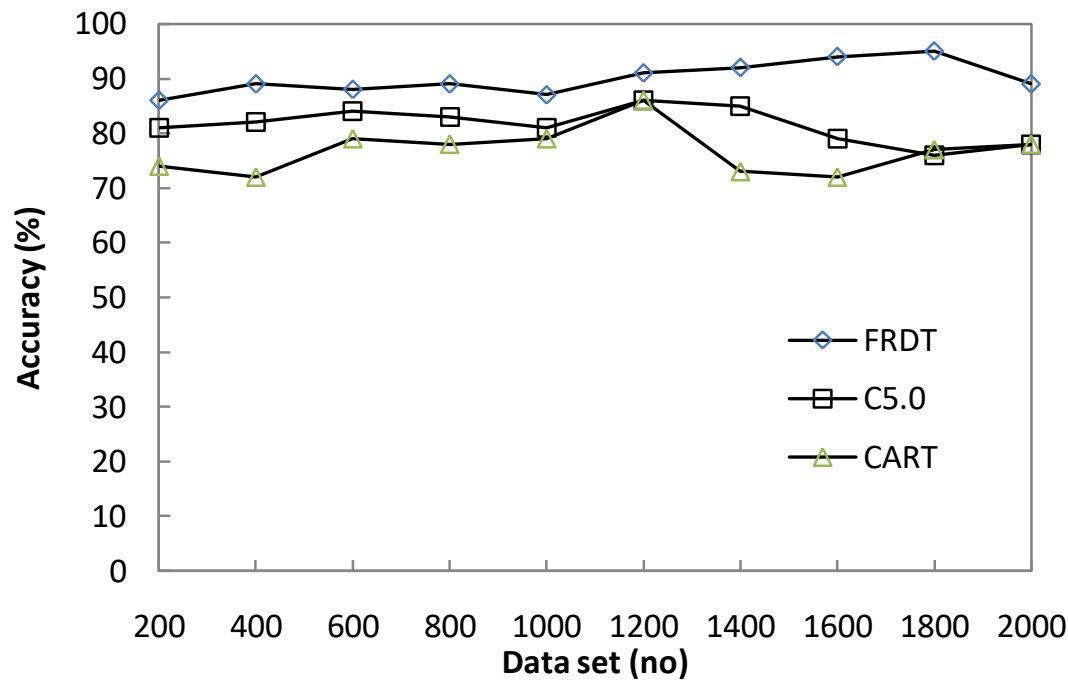

Fig.2 Accuracy

It very well may be seen from the Figure 2 that, since the model depends on this data set, the accuracy rate is very high, and the accuracy of certain data is near $100 \%$. Notwithstanding, with the expansion of data estimate, the accuracy rate has declined and has turned out to be insecure. The accuracy of the test data dependent on this
FRDT model is appeared in Figure 4. With reference to C5.0 and CART models are progressively accurate when the quantity of samples is large.Similarly error rate are appeared in fig 3. Which is unmistakably indicates thet FRDT give the most minimal error rate when contrast with the C5.0 and CART

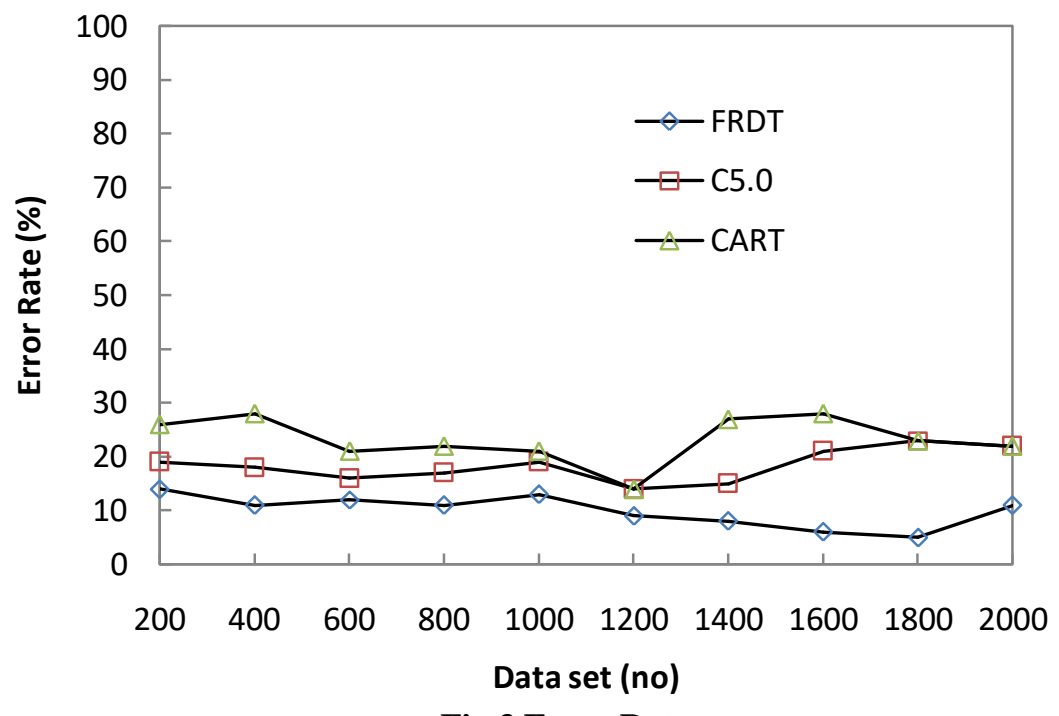

Fig.3 Error Rate 


\section{An Optimized Feature Regularization In Boosted Decision Tree}

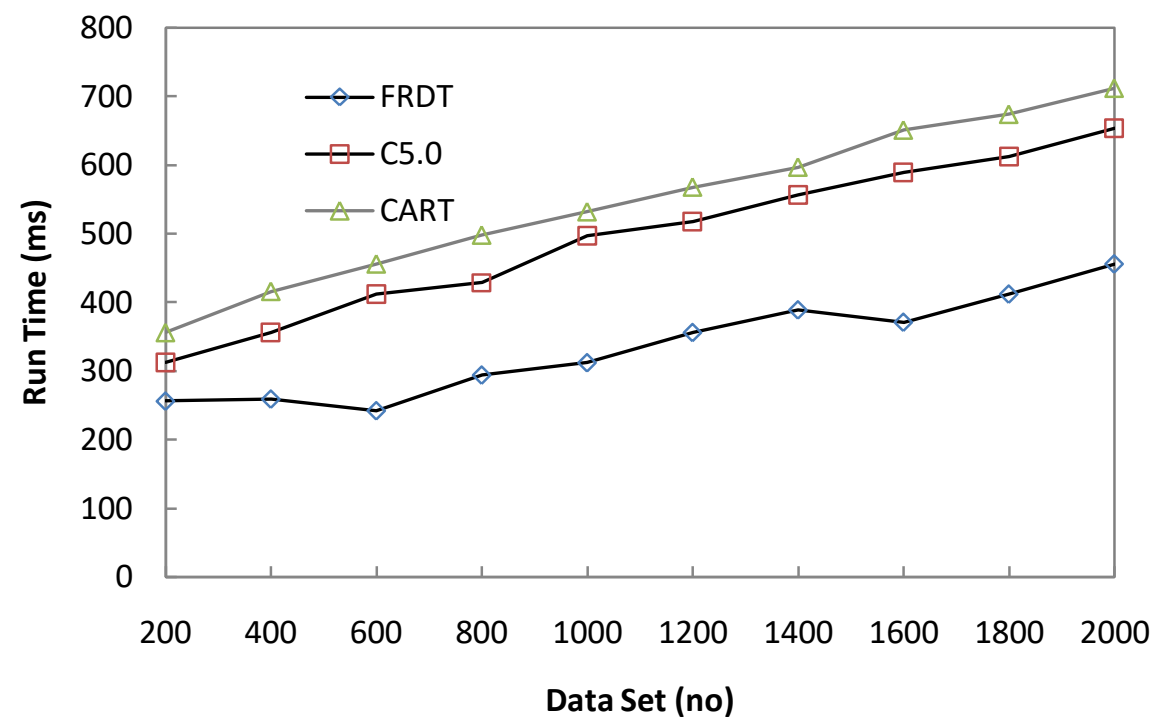

Fig.4 Runtime

The time curves of FRDT is linear, the inclines are little and the development is moderate. As the quantity of samples expands, the curve of the C5.0 strategy changes quicker, and the time is positively connected with the example measure. The curve of CART is on the ascent, and the pre-development rate is the fastest among the four strategies.

\section{CONCLUSION}

We have exhibited two feature selection strategies dependent on the SC basis and tentatively affirmed that they are a generally amazing option in contrast to the most well known techniques. On our way we have demonstrated a few instances of awesome improvement gotten by methods for feature selection. To decrease the intricacy of the scan for ideal feature sets we may discover a few regularities and use them as heuristics. Our methodology is based on a novel bound on classification or relapse error, ensuring that gains in speed don't come at a misfortune in classification execution. Examinations demonstrate that our strategy can decrease training cost by a request of size or more, or given a computational spending plan, can prepare classifiers that lessen errors by and large by two-overlay or more.

\section{REFERENCE}

1. K. Gr a abczewski. SSV criterion based discretization for Naive Bayes classifiers. In Proceedings of the 7th International Conference on Artificial Intelligence and Soft Computing, Zakopane, Poland, June 2004.

2. K. Gr abczewski and W. Duch. A general purpose separability criterion for classification systems. In Proceedings of the 4th Conference on Neural Networks and Their Applications, pages 203-208, Zakopane, Poland, June 1999.

3. K. Gr abczewski and N. Jankowski. Transformations of symbolic data for continuous data oriented models. In Artificial Neural Networks and Neural Information Processing - ICANN/ICONIP 2003, pages 359-366. Springer, 2003.

4. Guyon, A. Saffari, G. Dror, and G. Cawley, "Model selection: beyond the bayesian/frequentist divide," Journal of Machine Learning Research, vol. 11, pp. 61-87, 2010
5. H. Peng, F. Long, and C. Ding, "Feature selection based on mutual information: criteria of max-dependency, maxrelevance, and minredundancy," IEEE Transactions on Pattern Analysis and Machine Intelligence, vol. 27, no. 8, pp. 1226-1238, 2005

6. ] H. Liu and L. Yu, "Toward integrating feature selection algorithms for classification and clustering," IEEE Transactions on Knowledge and Data Engineering, vol. 17, no. 4, pp. 491-502, 2005

7. ] C. Aliferis, A. Statnikov, I. Tsamardinos, S. Mani, and X. Koutsoukos, "Local causal and markov blanket induction for causal discovery and feature selection for classification part i: Algorithms and empirical evaluation," Journal of Machine Learning Research, vol. 11, pp. 171-234, 2010

8. Svore, K. M. and Burges, C. J. Large-scale learning to rank using boosted decision trees. Scaling Up Machine Learning: Parallel and Distributed Approaches, 2011.

9. Viola, P. A. and Jones, M. J. Robust real-time face detection. International Journal of Computer Vision (IJCV), 2004.

10. Wu, J., Brubaker, S. C., Mullin, M. D., and Rehg, J. M. Fast asymmetric learning for cascade face detection. Pattern Analysis and Machine Intelligence (PAMI), 2008.

11. Doll'ar, P., Appel, R., and Kienzle, W. Crosstalk cascades for frame-rate pedestrian detection. In European Conference on Computer Vision (ECCV), 2012.

12. Domingo, C. and Watanabe, O. Scaling up a boostingbased learner via adaptive sampling. In Pacific-Asia Conference on Knowledge Discovery and Data Mining, 2000.

13. Domingos, P. and Hulten, G. Mining high-speed data streams. In International Conference on Knowledge Discovery and Data Mining, 2000.

14. Dubout, C. and Fleuret, F. Boosting with maximum adaptive sampling. In Neural Information Processing Systems (NIPS), 2011. 